\title{
The Coalescence Rate of Neutron Star-White Dwarf Binaries
}

\section{Nyadzani*}

Centre for Astro-Particle Physics (CAPP) and Department of Physics, University of Johannesburg, Auckland Park 2006, South Africa

E-mail: Inyadzani@uj.ac.za

\section{S. Razzaque}

Centre for Astro-Particle Physics (CAPP) and Department of Physics, University of Johannesburg, Auckland Park 2006, South Africa

E-mail: srazzaque@uj.ac.za

When two compact objects such as black holes (BH), white dwarfs (WD), and neutron stars (NS) orbit a common centre of gravity, they emit energy in the form of gravitational waves $(\mathrm{GW})$. The emission of GW will result in the two objects coalescing. In this work, we revisit the coalescence of NS-WD binary systems with the addition of one NS-WD binary system to the coalescing NSWD binary systems sample. The addition of this new system increased the coalescence rate from previously estimated $4.11_{-2.56}^{+5.25} \mathrm{Myr}^{-1}$ to $8.0_{-2.0}^{+24.0} \mathrm{Myr}^{-1}$ at the $90 \%$ confidence level.

7th Annual Conference on High Energy Astrophysics in Southern Africa - HEASA2019

28 - 30 August 2019

Swakopmund, Namibia

*Speaker. 


\section{Introduction}

The emission of gravitational waves from massive binaries was predicted by Albert Einstein. When two compact objects such as black holes (BH), white dwarfs (WD), and neutron stars (NS) orbit a common centre of gravity, they emit energy in the form of gravitational waves (GW) [1]. The emission of GW will result in the two objects coalescing. The study of the population of compact binary systems is important in understanding the emission of GWs. In this work we study the population of Neutron-Star White-Dwarf (NS-WD) binary systems. There are two ways in which one can study the population of compact binaries, one is by a theoretical approach using stellar evolution models and the second is by empirical calculations based on a sample of compact binary systems in our galaxy and statistics. In this work we use the empirical calculations to estimate the population and the merger rate of NS-WD systems.

This approach was used by Kim et al. in [2] in estimating the coalescence rate of NS-WD binaries following their work on the coalescence rate of double neutron star systems [3]. In [2] they use the properties of observed NS-WD binaries and Monte Carlo simulations to estimate the population of NS-WD systems with properties similar to the properties of the observed sample and the Galactic coalescence rate. In sampling the NS-WD binary systems one of the requirements to be counted as coalescing binary system is that a binary system must coalesce within the Hubble time $(t<10 G y r)$. During the time of their work, there were only 3 known coalescing NS-WD binaries in the Milky Way (PSR J0751 + 1807, PSR J1757 - 5322 and PSR J1141 - 6445). With these three systems, Kim et al. [2] found the coalescence rate $\left(\mathscr{R}_{t o t}\right.$ ) of NS-WD binaries to be $4.11_{-2.56}^{+5.25} \mathrm{Myr}^{-1}$ from their reference model at $68 \%$ statistical confidence limit. This rate of NS-WD binaries is low compared to the most recent coalescence rate of double neutron star (DNS) binaries of $42_{-14}^{+30} \mathrm{Myr}^{-1}$ at the 90\% confidence level [4].

Since the work of Kim et al. [2] more NS-WD systems have been discovered of which only one is a coalescing binary in the ATNF Pulsar Catalog [5]. In this work we follow the same approach as in [2] to estimate the Galactic coalescence rate of NS-WD binary systems with the addition of one coalescing NS-WD system: PSR J1952 + $2630[5,6]$.

In section 2, we show how we calculate the life-time of a binary system for population simulations matching pulsar surveys and tabulate the properties of all known coalescing NS-WD binaries in our Galaxy. In sections 3, we give a brief description of the statistical methodology presented in [3] used in calculating the population distribution and the rates. In section 4, we describe the survey simulation, list all the pulsar surveys considered in this work and and finally present our Galactic coalescence rate in section 5 .

\section{Coalescing NS-WD Binaries}

There are more than 2000 known neutron stars in binary systems in the ATNF Pulsar Catalog . These neutron stars are in binary systems with either a planet, main sequence star, neutron star, or a white dwarf [5]. About 100 of these neutron stars have a white dwarf as a companion and only 4 of these are coalescing systems. For coalescence we require that a binary system merges within the Hubble time $\left(\tau_{m r g}<10 \mathrm{Gyr}\right)$ due to the emission of GW. The coalescence rate of binary systems is given by the ratio between the number of binary systems and their life time. There are 
some uncertainties in estimating the life time of binary systems and this affect the coalescence rate of binary systems (see [7] for detailed discussion). In this work we follow the procedure of [2] when estimating the life time of a a binary system in order to simulate a population of binaries with observed properties of a survey. We take the life time of a binary system to be the sum of the current age $\left(\tau_{\text {age }}\right)$ and the remaining life time of the system $\tau_{\text {obs }}$,

$$
\tau_{\text {life }}=\tau_{\text {age }}+\tau_{\text {obs }}
$$

In calculating the current age of the pulsar we take the minimum between the characteristic age of the pulsar and the current "spin-down" age and for the remaining observable life of the system we take the minimum between the time to merger due to the emission of GW and the pulsar death time.

$$
\tau_{\text {life }}=\min \left(\tau_{c}, \tau_{s d}\right)+\min \left(\tau_{m r g}, \tau_{d}\right)
$$

here $\tau_{c}$ is the characteristic age of the pulsar, $\tau_{s d}$ is the current "spin-down" age, $\tau_{m r g}$ is the coalescence time of the binary system due to the emission of gravitational waves [8] and $\tau_{d}$ is the pulsar death time.

$$
\tau_{c}=\frac{P}{2 \dot{P}}
$$

where $P$ is the current period of the pulsar and $\dot{P}$ is the period derivative and

$$
\tau_{s d}=\tau_{c}\left(1-\left[\frac{P_{0}}{P}\right]^{2}\right)
$$

where $P_{0}$ is the birth period of the pulsar and assuming that the spin-down rate has not changed since birth, we adopt the spin-up line from [9] and write the birth period as,

$$
P_{0}=\left(\frac{\dot{P} P}{1.1 \times 10^{-15} \mathrm{~s}^{-4 / 3}}\right)^{3 / 7}
$$

and the pulsar death time is given by,

$$
\tau_{d}=\left(\frac{P_{d}^{2}-P^{2}}{2 P \dot{P}}\right)
$$

here $P_{d}$ is the pulsar "death-period" given by

$$
P_{d}=\left(\frac{B_{s}}{1.4 \times 10^{11} G}\right)^{7 / 13} \mathrm{~s}
$$

and $B_{s}$ is the surface magnetic field of the neutron star and can be estimated from the current spin period $P(\mathrm{~s})$ and the spin-down rate $\dot{P}\left(\mathrm{ss}^{-1}\right)[10]$,

$$
B_{s}=3.2 \times 10^{19}(P \dot{P})^{1 / 2} G .
$$

The merger time $\tau_{m r g}$ is calculated using an analytical solution we developed in [11] following Peters [8]. The four known NS-WD coalescing systems and their orbital properties in Table 1. Note that for J0751+1807, J1952+2630 and J1757-5322; $\tau_{m r g}<\tau_{d}$ and the lifetime is calculated as $\tau_{\text {life }}=\tau_{s d}+\tau_{m r g}$. Only for J1141-6545; $\tau_{m r g}>\tau_{d}$ and the lifetime is $\tau_{\text {life }}=\tau_{c}+\tau_{d}$ [2]. 
Table 1: List of coalescing NS-WD systems in the Milky Way from the ATNF Pulsar Catalog [5]. $P$ is the pulsar period, $\dot{P}$ is the period derivative, $P_{b}$ is the binary period, $\tau_{d}$ is the observable lifetime. The big mass is of a NS $\left(m_{N S}\right)$ and small mass is of WD $\left(m_{W D}\right)$.

\begin{tabular}{|c|c|c|c|c|c|c|c|c|c|c|c|}
\hline $\begin{array}{l}\text { Name } \\
\text { (PSR }\end{array}$ & $\begin{array}{l}P \\
(\mathrm{~ms})\end{array}$ & $\begin{array}{l}\dot{P} \\
(s / s) \\
\end{array}$ & $\begin{array}{l}P_{0} \\
\mathrm{~s}\end{array}$ & $\begin{array}{l}P_{b} \\
(\mathrm{hr})\end{array}$ & $\begin{array}{l}\text { Masses } \\
\left(M_{\odot}\right) \\
\end{array}$ & $\mathrm{e}$ & $\begin{array}{l}\tau_{c} \\
(\mathrm{Gyr})\end{array}$ & $\begin{array}{l}\tau_{s d} \\
(\mathrm{Gyr})\end{array}$ & $\begin{array}{l}\tau_{d} \\
(\mathrm{Gyr})\end{array}$ & $\begin{array}{l}\tau_{m r g} \\
(\mathrm{Gyr}) \\
\end{array}$ & Ref \\
\hline $\mathrm{J} 0751+1807$ & 3.48 & $8.01 \cdot 10^{-21}$ & $5.57 \cdot 10^{-4}$ & 6.32 & $\begin{array}{l}2.20 \\
0.18\end{array}$ & $3 \cdot 10^{-6}$ & 6.8 & 6.7 & 401 & 7.6 & {$[12,13]$} \\
\hline $\mathrm{J} 1952+2630$ & 20.73 & $4.27 \cdot 10^{-18}$ & $1.76 \cdot 10^{-2}$ & 9.40 & $\begin{array}{l}1.40 \\
0.95\end{array}$ & $4 \cdot 10^{-5}$ & 0.076 & 0.037 & 9.8 & 3.9 & [6] \\
\hline $\mathrm{J} 1141-6545$ & 393.9 & $4.29 \cdot 10^{-15}$ & 1.20 & 4.74 & $\begin{array}{l}1.43 \\
0.99\end{array}$ & 0.172 & 0.0015 & $\ldots$ & 0.104 & 0.6 & [14] \\
\hline $\mathrm{J} 1757-5322$ & 8.87 & $2.78 \cdot 10^{-15}$ & $1.41 \cdot 10^{-3}$ & 10.88 & $\begin{array}{l}1.35 \\
0.67\end{array}$ & $10^{-6}$ & 5.1 & 4.9 & 145 & 7.8 & [15] \\
\hline
\end{tabular}

\section{Statistical Analysis}

Here we present the statistical analysis developed by Kim et al. in [3]. For a fixed value of $N_{t o t}$, the observed samples is used to estimate the distribution of objects with like properties. One might expect that the number of observed pulsars in a sample $N_{o b s}$ follows very closely a Poisson distribution:

$$
P\left(N_{o b s} ; \lambda\right)=\frac{\lambda^{N_{o b s}} e^{-\lambda}}{N_{o b s} !}
$$

where, by definition $\lambda \equiv\left\langle N_{o b s}\right\rangle$. Following arguments presented in [3], the relationship between $\lambda$ and $N_{\text {tot }}$ is linear,

$$
\lambda=\alpha N_{\text {tot }}
$$

where $\alpha$ is a constant that depends on the properties of the Galactic pulsar population and the Pulsar surveys given by $\alpha=1 / N_{\text {tot }}$. The probability distribution of the total number of pulsars with properties of the observed sample is obtained by applying Baye's theorem,

$$
P(H \mid D X)=P(H \mid X) \frac{P(D \mid H X)}{P(D \mid X)}
$$

here $H$ is our model hypothesis $\lambda$, which is proportional to $N_{\text {tot }}$ (see equation (3.2)) $D$ is the real observed sample, $X$ is the population model. In equation (3.3), $P(H \mid D X)$ is the probability of a model hypothesis $H$ given data $D$ and model priors $X, P(H \mid X)$ is the probability of a model hypothesis without any data, $P(D \mid H X)$ is the likelihood of the data given a model hypothesis $H$ and priors $X$, and $P(D \mid X)$ is the model prior probability, this acts as a normalization constant. The likelihood of the real observed sample $P(D \mid H X)$ (with $D=1$ ) is given by the best fitting Poisson distribution,

$$
P(D \mid H X)=P\left[1 ; \lambda\left(N_{t o t}\right), X\right]=\lambda\left(N_{t o t}\right) e^{-\lambda\left(N_{t o t}\right)}
$$


without any data information, given a set of assumptions $X$,

$$
P\left[\lambda\left(N_{t o t}\right) \mid X\right]=\text { constant }
$$

hence

$$
P(\lambda \mid D X)=\text { constant } \cdot P\left(1 ; \lambda\left(N_{t o t}\right), X\right)
$$

Note the normalization constant $P(D \mid X)$ is absorbed by the constant $P\left[\lambda\left(N_{t o t}\right) \mid X\right]$. The normalization constraint $\int_{0}^{\infty} P(\lambda \mid D X) d \lambda=1$ gives, $P(H \mid X) / P(D \mid X)=$ constant $=1$, then

$$
P(1 ; \lambda, X)=P(\lambda)=\lambda e^{-\lambda}
$$

Note the maximum value of $P(\lambda)$ is $P(1)=1 / e$. With $P(\lambda)$ we calculate $P\left(N_{t o t}\right)$ using equation (3.2) as follow,

$$
\begin{aligned}
P\left(N_{t o t}\right) & =P(\lambda)\left|\frac{d \lambda}{d N_{t o t}}\right| \\
& =\alpha^{2} N_{t o t} e^{-\alpha N_{t o t}}
\end{aligned}
$$

The coalescence rate of a binary system containing an observable radio pulsar is given by,

$$
\mathscr{R}=\frac{N_{\text {tot }}}{\tau_{\text {life }}} \times f_{b}
$$

here, $N_{t o t}$ is the estimated number of pulsars in our Galaxy with pulse profiles and orbital characteristics similar to those of known systems, and $f_{b}$ is a correction factor for pulsar beaming. This correction factor $\left(f_{b}\right)$ is needed to account for the fraction of pulsars that remain undetected not because of survey selection effects but because they do not beam in our direction [16,17]. The correction factor depends on the distribution of pulsar beam sizes which may be different for different types of pulsars. Taking into account the half-opening angle $(\alpha)$ in the latitude direction and the magnetic inclination angle $(\rho)$ (relative to the spin axis), the beaming fraction is given by [7],

$$
f_{b}=\left[\frac{2}{2 \pi} \int_{0}^{2 \pi} d \phi \int_{\alpha-\rho}^{\alpha+\rho} \sin \theta d \theta\right]^{-1}
$$

Then the Galactic merger rate probability distribution is calculated using,

$$
\begin{aligned}
P(\mathscr{R}) & =P\left(N_{\text {tot }}\right)\left|\frac{d N_{\text {tot }}}{d \mathscr{R}}\right| \\
& =\left(\frac{\alpha \tau_{\text {life }}}{f_{d}}\right)^{2} \mathscr{R} e^{-\left(\alpha \tau_{\text {life }} \mathscr{R} / f_{b}\right)}
\end{aligned}
$$


and the Milky Way merger rate will be the sum of all individual rates calculated in equation (3.9) and is given by,

$$
\mathscr{R}_{M W}=\sum_{n=1}^{m} \mathscr{R}_{n}
$$

The Galactic merger rate probability distribution is given by a convolution of the individual merger rate probability distributions in equation (3.11) as follow,

$$
P\left(\mathscr{R}_{M W}\right)=\prod_{n=1}^{m} P\left(\mathscr{R}_{n}\right)
$$

\section{Population simulations}

We make use of the freely available PsrPopPy ${ }^{1}$ software to model the pulsar population and survey selection effects [18]. Our pulsar surveys are selected in such a way that they cover as much sky as possible. We simulate the following pulsar surveys in this work, Parkes multi-beam survey [19], PALFA Galactic plane survey at full resolution [20], The Parkes High-Latitude Pulsar Survey [21], Putative pulsar survey with LOFAR [22], HTRU [23]. From these simulation we obtained the number of pulsars $N_{P S R}$ in our Galaxy with pulse profiles and orbital characteristics similar to those of the known systems in Table 1 . We do not include the beaming corrections in our calculation of coalescence rate for the same reasons mentioned in [2], hence we let $f_{b}=1$.

Table 2: Results from simulation showing the estimated number of pulsars in our Galaxy with pulse profiles and orbital characteristics similar to those of known systems $N_{P S R}$ and their life time.

\begin{tabular}{l|l|l}
\hline \hline PSR & $N_{P S R}$ & $\tau_{\text {life }}(\mathrm{Gyr})$ \\
\hline $\mathrm{J} 0751+1807$ & 1700 & 14.3 \\
$\mathrm{~J} 1952+2630$ & 1220 & 12.7 \\
$\mathrm{~J} 1141-6545$ & 680 & 0.105 \\
$\mathrm{~J} 1757-5322$ & 1430 & 3.93 \\
\hline \hline
\end{tabular}

\section{Results and Discussion}

The coalescence rates for each of the known individual binary systems in table 2 are calculated using equation (3.11) and their probability distribution is shown in Figure 1. PSR J1141 - 6545 has the highest coalescence rate followed by the newly added PSR J1952 +2630 . Even though PSR J0751 + 1807 has the highest population its lifetime is very high, and it therefore has a low coalescence rate. The small lifetime of PSR J1141 - 6545 means it has high merging rate. The individual merger rates peak at $6.48_{-1.43}^{+14.75} \mathrm{Myr}^{-1}, 0.12_{0.05}^{+0.49} \mathrm{Myr}^{-1}, 0.09_{-0.02}^{+0.22} \mathrm{Myr}^{-1}$, and $0.49_{-0.13}^{+1.13}$ $\mathrm{Myr}^{-1}$ for PSR J1141 - 6545, PSR J0751 + 1807 , PSR J1757 - 5322, PSR J1952 + 2630, and respectively.

\footnotetext{
${ }^{1}$ https://github.com/samb8s/PsrPopPy
} 


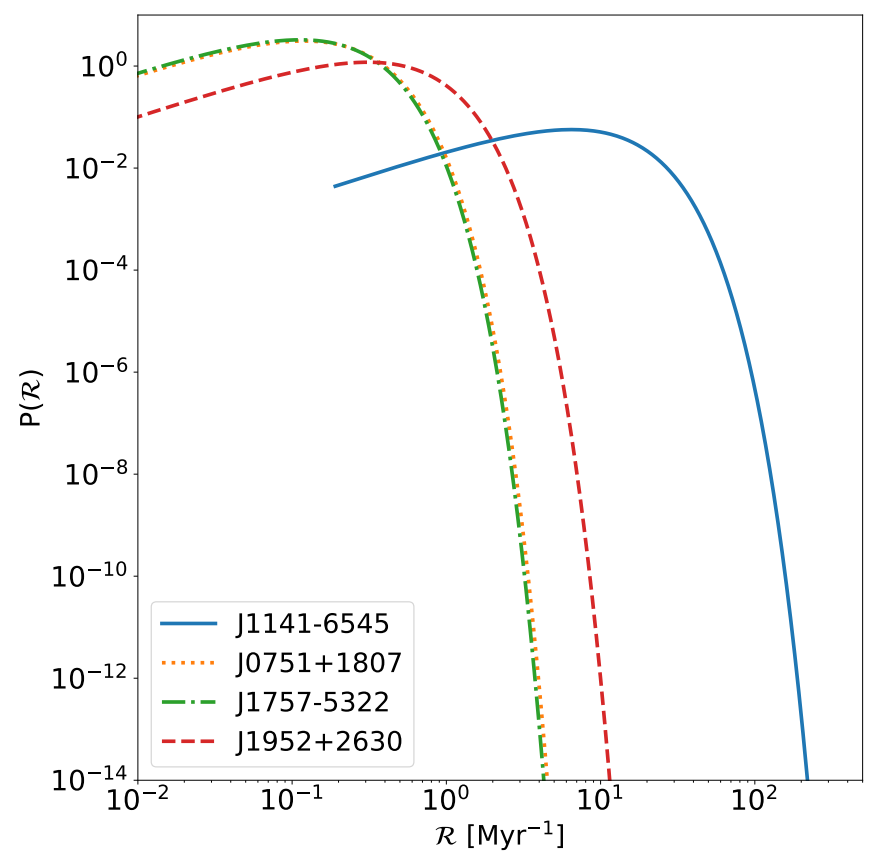

Figure 1: This figure shows the probability of the individual coalescence rates of the four coalescing NSWD binaries in our galaxy. The probability for PSR J1952 + 2630 is represented by dashed line (red), PSR J1757 - 5322 by dash-dot (green) line, PSR J0751 + 1807 by dot (orange) line and PSR J1141 - 6545 by solid (blue) line.

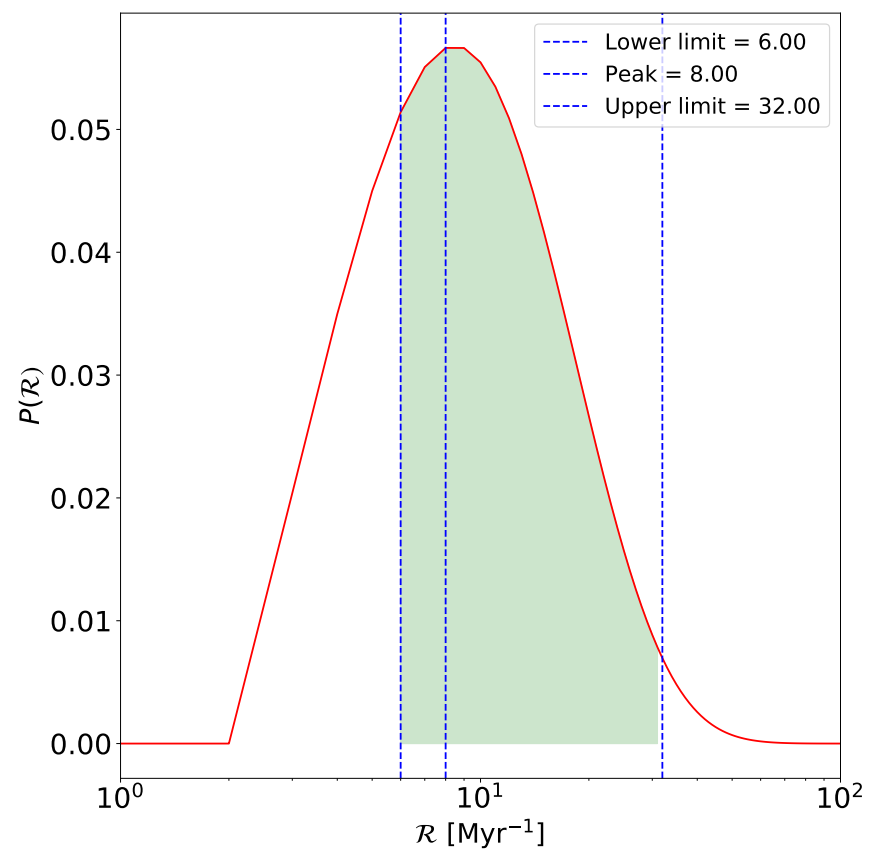

Figure 2: The probability distribution of the Galactic coalescence rate at $90 \%$ confidence level. 
Figure 2 shows the Galactic rate is calculated by summing the individual rates using equation (3.13) where $m=4$. There is an increase in the Galactic rate from $4.11_{-2.56}^{+5.25} \mathrm{Myr}^{-1}$ [2] to $8.0_{-2.0}^{+24.0}$ $\mathrm{Myr}^{-1}$ according to our calculation. The newly added NS-WD system PSR J1952 + 2630 contributes more to the Galactic rate than PSR J1757 - 5322 and PSR J0751 + 1807 combined.

\section{Conclusions}

The coalescence rate of NS-WD systems increased with the addition of PSR J1952 + 2630 . PSR J1952 + 2630 contributes more than PSR J1757 - 5322 and PSR J0751 + 1807 combined to the Galactic rate. High sensitivity surveys (e.g. with MeerKAT/SKA) can help to constrain the NS-WD population in the Milky Way and the beaming correction would decrease the uncertainty on the coalescence rate.

\section{Acknowledgement}

This research was supported by the South African Gamma-ray Astronomy Programme (SA-GAMMA) and a CPRR grant (No. 111749) of the National Research Foundation (NRF) to S.R.

\section{References}

[1] A. Einstein, Über gravitationswellen, Sitzungsberichte der Königlich Preußischen Akademie der Wissenschaften (Berlin), Seite 154-167. (1918) .

[2] C. Kim, V. Kalogera, D. R. Lorimer and T. White, The probability distribution of binary pulsar coalescence rates. ii. neutron star-white dwarf binaries, The Astrophysical Journal 616 (2004) 1109.

[3] C. Kim, V. Kalogera and D. Lorimer, The probability distribution of binary pulsar coalescence rates. i. double neutron star systems in the galactic field, The Astrophysical Journal 584 (2003) 985.

[4] N. Pol, M. McLaughlin and D. R. Lorimer, Future prospects for ground-based gravitational-wave detectors: The galactic double neutron star merger rate revisited, The Astrophysical Journal $\mathbf{8 7 0}$ (2019) 71.

[5] R. N. Manchester, G. B. Hobbs, A. Teoh and M. Hobbs, The australia telescope national facility pulsar catalogue, The Astronomical Journal 129 (2005) 1993, http://www.atnf.csiro.au/research/pulsar/psrcat.

[6] B. Knispel, P. Lazarus, B. Allen, D. Anderson, C. Aulbert, N. Bhat et al., Arecibo palfa survey and einstein@ home: binary pulsar discovery by volunteer computing, The Astrophysical Journal Letters 732 (2011) L1.

[7] V. Kalogera, R. Narayan, D. Spergel and J. Taylor, The coalescence rate of double neutron star systems, The Astrophysical Journal 556 (2001) 340.

[8] P. C. Peters, Gravitational radiation and the motion of two point masses, Physical Review 136 (1964) B1224.

[9] Z. Arzoumanian, J. Cordes and I. Wasserman, Pulsar spin evolution, kinematics, and the birthrate of neutron star binaries, The Astrophysical Journal 520 (1999) 696. 
[10] K. Chen and M. Ruderman, Pulsar death lines and death valley, The Astrophysical Journal 402 (1993) 264-270.

[11] L. Nyadzani and S. Razzaque, An analytical solution to the coalescence time of compact binary systems, arXiv preprint arXiv:1905.06086 (2019) .

[12] D. J. Nice, E. M. Splaver and I. H. Stairs, Heavy neutron stars? a status report on arecibo timing of four pulsar-white dwarf systems, .

[13] S. Lundgren, A. Zepka and J. Cordes, A millisecond pulsar in a 6 hour orbit: Psr j0751+ 1807, The Astrophysical Journal 453 (1995) 419.

[14] R. T. Edwards and M. Bailes, Discovery of two relativistic neutron star-white dwarf binaries, The Astrophysical Journal Letters 547 (2001) L37.

[15] M. Bailes, S. Ord, H. Knight and A. Hotan, Self-consistency of relativistic observables with general relativity in the white dwarf-neutron star binary psr j1141-6545, The Astrophysical Journal Letters 595 (2003) L49.

[16] R. Narayan, T. Piran and A. Shemi, Neutron star and black hole binaries in the galaxy, The Astrophysical Journal 379 (1991) L17-L20.

[17] E. S. Phinney, The rate of neutron star binary mergers in the universe-minimal predictions for gravity wave detectors, The Astrophysical Journal 380 (1991) L17-L21.

[18] S. D. Bates, D. R. Lorimer, A. Rane and J. Swiggum, PsrPopPy: an open-source package for pulsar population simulations, Monthly Notices of the Royal Astronomical Society 439 (02, 2014) 2893-2902.

[19] R. N. Manchester, A. G. Lyne, F. Camilo, J. Bell, V. Kaspi, N. D’Amico et al., The parkes multi-beam pulsar survey-i. observing and data analysis systems, discovery and timing of 100 pulsars, Monthly Notices of the Royal Astronomical Society 328 (2001) 17-35.

[20] J. M. Cordes, P. Freire, D. R. Lorimer, F. Camilo, D. J. Champion, D. J. Nice et al., Arecibo pulsar survey using alfa. i. survey strategy and first discoveries, The Astrophysical Journal 637 (2006) 446.

[21] M. Burgay, B. Joshi, N. D’Amico, A. Possenti, A. Lyne, R. Manchester et al., The parkes high-latitude pulsar survey, Monthly Notices of the Royal Astronomical Society 368 (2006) 283-292.

[22] S. Bates, S. Johnston, D. Lorimer, M. Kramer, A. Possenti, M. Burgay et al., A 6.5-ghz multibeam pulsar survey, Monthly Notices of the Royal Astronomical Society 411 (2011) 1575-1584.

[23] M. Keith, A. Jameson, W. Van Straten, M. Bailes, S. Johnston, M. Kramer et al., The high time resolution universe pulsar survey-i. system configuration and initial discoveries, Monthly Notices of the Royal Astronomical Society 409 (2010) 619-627. 